\title{
Variaciones temporales de las comunidades de parásitos en peces intermareales de Chile central: hospedadores residentes $v s$ temporales
}

Temporal variations in the parasite communities of intertidal fish from central Chile: resident vs temporal hosts

\section{Gabriela Muñoz ${ }^{1}$ y Natalí Delorme ${ }^{2}$}

\author{
${ }^{1}$ Facultad de Ciencias del Mar y de Recursos Naturales, Universidad de Valparaíso, Casilla 5080, Reñaca, Viña del Mar, Chile. \\ gabriela.munoz@cienciasdelmar.c1 \\ ${ }^{2}$ School of Biological Sciences, University of Auckland, Private Bag 92019, Auckland 1142, New Zealand
}

\begin{abstract}
This study determines the existence of temporal patterns in parasite communities of resident (Scartichthys viridis and Helcogrammoides chilensis) and temporal fishes (Girella laevifrons and Sicyases sanguineus) from the rocky intertidal of central Chile. Fish were seasonally collected, from winter 2006 to winter 2009. In resident fishes, the mean infracommunity abundance and richness, as well as the total prevalence of parasites, varied seasonally during the three years. The highest values of total prevalence, abundance and richness of parasites were observed during the spring-summer periods, whereas the lowest values were found during the autumn-winter periods. However, the magnitude of these community descriptors differed among years. For temporal fishes, there were great variations within the parasite community descriptors, with little congruence among years. Part of this variation in both fish groups was because of the fish total length (TL), as there were positive and significant correlations between parasitological descriptors and fish TL; however, its influence was clearly seasonally demonstrated only in S. viridis, whereas the TL of the other fish species was less or not important for the variation in the abundance, richness and prevalence of parasites. It is possible that other factors, such as environmental characteristics, host abundances (intermediate or definitive hosts), and the type and timing of parasite life cycles might have different influences in the temporal variation of their communities, showing the temporal patterns seen in this research.
\end{abstract}

Key words: Metazooan parasites, seasonal patterns, parasites' infracommunities

Resumen.- Este estudio determina la existencia de patrones temporales en las comunidades de parásitos de peces residentes (Scartichthys viridis y Helcogrammoides chilensis) y temporales (Girella laevifrons y Sicyases sanguineus) del intermareal rocoso de Chile central. Los peces fueron obtenidos estacionalmente, desde el invierno 2006 hasta el invierno 2009. La abundancia y riqueza infracomunitaria promedio así como la prevalencia total de parásitos fue variable en el tiempo; en peces residentes se observó patrones de variación estacional relativamente consistentes ente un año y otro, con valores máximos de prevalencia, abundancia y riqueza de parásitos en primavera-verano, y valores bajos en otoño-invierno. No obstante, la magnitud de estos descriptores comunitarios fue distinta entre un año y otro. En peces temporales se observó alta variación en los descriptores comunitarios de parásitos, con poca consistencia entre años. Parte de las variaciones comunitarias de parásitos observadas en ambos grupos de peces, se debió a la longitud total (LT) de los peces, ya que en general hubo correlaciones positivas y significativas entre los descriptores parasitológicos y la LT. Sin embargo, su influencia estacional solo se evidenció en S. viridis, mientras que para otras especies de peces la LT fue poco o nada de importante para las variaciones de abundancia, riqueza y prevalencia de parásitos. Es posible que otras variables, tales como factores ambientales, abundancia de hospedadores (intermediarios o definitivos), o tipos y tiempos de los ciclos de vida de los parásitos, tengan distintas influencias en las variaciones de sus comunidades, derivando en los patrones temporales aquí observados.

Palabras clave: Parásitos metazoos, patrones estacionales, infracomunidades de parásitos 


\section{INTRODUCCIÓN}

Las dinámicas poblacionales y comunitarias de los organismos pueden estar modeladas por distintas características ambientales que varían en escalas espaciotemporales, como respuesta a cambios de los factores físico-químicos de su entorno, recursos básicos y la presencia de otras especies, como competidores y depredadores. Estos factores cambian cíclicamente en el tiempo, o abruptamente en otros casos, lo que afecta en la variación de la abundancia, distribución espacial y reproducción de los organismos a lo largo del tiempo y el espacio (e.g., Flecker \& Feifarek 1994, Hewitt et al. 2001).

Las poblaciones y comunidades de parásitos también responden a los cambios ambientales, y por tanto, pueden presentar patrones de variación temporal (e.g., Fernández \& Esch 1991, Zander 2003). Tanto las características biológicas del hospedador (microambiente) como el ambiente externo al hospedador (macroambiente del parásito) constituyen los factores principales que estructuran y causan cambios en las poblaciones y comunidades de parásitos (e.g., Inostroza et al. 1993, Zander et al. 1999, Sandland et al. 2001, Marcogliese 2002, Poulin 2007).

El patrón de variación temporal de las comunidades de parásitos puede ser mensual, estacional o interanual, dependiendo de los cambios de diversos factores y su efecto en los parásitos. Usualmente, las investigaciones están dirigidas a las variaciones estacionales de poblaciones de parásitos, dado que éstos pueden asociarse a cambios ambientales y biológicos del hospedador (Granath \& Esch 1983, Zander 2003, González \& Oliva 2006, Herrmann \& Sorensen 2009). Otros estudios, en cambio, han determinado que las comunidades de parásitos de organismos acuáticos varían poco (en composición y descriptores) entre años consecutivos (e.g., Díaz \& George-Nascimento 2002, Cortés \& Muñoz 2009). Esto se debe a que un ambiente tiende a variar natural y similarmente entre un año y otro, mientras que entre varios años de diferencias, pueden ocurrir importantes cambios en el ecosistema, repercutiendo en los ciclos de vida, la infectividad o la sobrevivencia de los parásitos (e.g., Hurtrez-Boussès et al. 1999, Fellis \& Esch 2004, Yurlova et al. 2006, Negovetich \& Esch 2007, Cortés \& Muñoz 2009, Herrmann \& Sorensen 2009, Kim \& Powell 2009).

Los peces que habitan en la zona intermareal son los vertebrados más abundantes y frecuentes de este ambiente (Horn \& Gibson 1988). Estos peces, según su especie, tienen distintos tiempos de permanencia en la zona intermareal, distinguiéndose dos grupos: los peces temporales, aquellos que viven en esa zona solo por un período corto de tiempo asociado a un determinado estadio ontogénico (juveniles o adultos), y los peces residentes, aquellos que viven permanentemente en el ambiente intermareal encontrándose tanto adultos como juveniles (Varas \& Ojeda 1990, Gibson \& Yoshiyama 1999). Se ha demostrado que ambos grupos de peces, residentes y temporales, poseen diferencias en la composición y carga parasitaria; peces temporales y en estadio juvenil, suelen tener menor abundancia y riqueza de parásitos que los peces residentes (Muñoz \& Cortés 2009).

Dentro de las especies de peces residentes del intermareal rocoso de Chile central se encuentran Scartichthys viridis (Valenciennes, 1836) (Blenniidae) y Helcogrammoides chilensis (Cancino, 1960) (Trypterigidae) las cuales son especies abundantes y persistentes en las pozas intermareales durante todo el año aunque presentan distinto tamaño corporal entre sí (Muñoz \& Ojeda 1998); S. viridis alcanza hasta $45 \mathrm{~cm}$ de longitud total (LT) (Muñoz-Muga \& Muñoz 2010), mientras que $H$. chilensis puede alcanzar hasta $7,8 \mathrm{~cm}$ (Muñoz \& Ojeda 1998). Por su parte, las especies de peces temporales más abundantes y en estadio juvenil en la zona intermareal del Chile central son Girella laevifrons (Tschudi, 1846) (Kyphosidae) y Sicyases sanguineus Müller \& Troschel, 1843 (Gobiesocidae) (Muñoz \& Cortés 2009). Los juveniles de estas especies tienen tamaños que oscilan entre 2 y $20 \mathrm{~cm}$ de LT aproximadamente, mientras que los adultos pueden alcanzar 30 a $35 \mathrm{~cm}$ de LT (Mann 1954, Muñoz \& Zamora 2011). Con respecto los antecedentes parasitológicos, existen descripciones de las infracomunidades de parásitos para S. viridis (Díaz \& George-Nascimento 2002, Muñoz-Muga \& Muñoz 2010, Muñoz \& Randhawa 2011) y S. sanguineus (Muñoz \& Zamora 2011), mientras que para G. laevifrons y $H$. chilensis existen solo registros del número de especies parásitas, aunque sin referencia de sus identidades específicas (Aldana et al. 2002, Muñoz \& Cortés 2009). Sólo $S$. viridis cuenta con estudios de variación estacional de sus comunidades de parásitos (Muñoz \& Randhawa 2011).

Existen antecedentes que permiten hipotetizar que las comunidades de parásitos varían de forma distinta entre los peces residentes y temporales, debido a que ambos grupos de peces tienen distintos tiempos de permanencia en el intermareal y además, se encuentran en distintos estadios de desarrollo. Por un lado, la riqueza de especies 
y la abundancia de parásitos dentro de una misma especie hospedadora suele ser menor en peces juveniles que en adultos (Muñoz et al. 2002, Muñoz \& Zamora 2011), mostrando una relación positiva de la carga y la riqueza parasitaria con respecto al tamaño corporal y edad del hospedador. Por otro lado, los peces juveniles temporales han presentado distinta composición parasitara aún cuando provienen de localidades cercanas (Muñoz et al. 2001, 2002), lo cual indica que sus comunidades parásitas pueden ser altamente variables. En peces residentes del intermareal, como por ejemplo Scartichthys viridis, las comunidades de parásitos son estables entre años consecutivos (Díaz \& George-Nascimento 2002) y presentan un patrón de variación estacional (Muñoz \& Randhawa 2011). Por lo expuesto, este estudio tuvo como objetivos, 1) determinar si existen variaciones en las comunidades de parásitos en las 4 especies de peces intermareales mencionadas anteriormente, entre año y entre estaciones, y 2) determinar si existen diferencias en los patrones de variación comunitaria de parásitos entre peces residentes y temporales.

\section{Materiales Y MÉTOdos}

Los peces fueron recolectados desde pozas intermareales rocosas de Isla Negra ( $\left.33^{\circ} 15^{\prime} \mathrm{S}, 71^{\circ} 30^{\prime} \mathrm{O}\right)$, El Tabo (33⒉ $27^{\prime} \mathrm{S}$, $\left.71^{\circ} 38^{\prime} \mathrm{O}\right)$ y Las Cruces ( $\left.33^{\circ} 29^{\prime} \mathrm{S}, 71^{\circ} 37^{\prime} \mathrm{O}\right)$, Chile central. La captura de peces se realizó durante la baja marea con la aplicación de anestésico BZ-20 $0^{\circledR}$ y redes de mano, entre invierno 2006 e invierno 2009, obteniéndose 13 muestras temporales para cada especie de pez. De este modo, se obtuvieron 3 periodos anuales; Año 1: invierno 2006 a otoño 2007, Año 2: invierno 2007 a otoño 2008; Año 3: invierno 2008 a otoño 2009. Las muestras de invierno 2009 fueron solo consideradas para los descriptores parasitológicos generales, pero no en las comparaciones estadísticas entre años y estaciones.

En total se recolectaron 3.351 ejemplares. En peces residentes se obtuvieron 826 ejemplares de Helcogrammoides chilensis y 1.475 de Scartichthys viridis, y en peces temporales se obtuvieron 500 ejemplares de Girella laevifrons y 550 de Sicyases sanguineus. Al momento de la captura, los peces fueron depositados individualmente en bolsas plásticas y posteriormente llevados al laboratorio. La mayoría de los peces fueron congelados a $-10^{\circ} \mathrm{C}$ para su posterior examen, mientras que otros fueron disectados en fresco con el propósito de obtener parásitos en buenas condiciones para procesos de tinción y de identificación.

Cada pez fue medido en longitud total (LT) y disectado para la búsqueda y recolección de sus parásitos. Los ectoparásitos fueron recolectados desde la cavidad bucal, branquias, piel, aletas y canales operculares; mientras que los endoparásitos fueron recolectados desde la cavidad celomática, tracto digestivo y musculatura. Los parásitos obtenidos fueron fijados y almacenados en formalina al $10 \%$ para su posterior determinación taxonómica. Para la identificación de parásitos, se realizaron preparaciones in toto, con tinción de hematoxilina, para tremátodos y monogeneos. Copépodos y nemátodos fueron transparentados con lactofenol de Amman. Los parásitos fueron identificados según la literatura citada en Muñoz \& Cortés (2009).

Para cada especie de pez se describió su comunidad de parásitos mediante el cálculo del promedio de abundancia e intensidad infrapoblacional, y la prevalencia de cada especie parásita (Bush et al. 1997). Para el caso de las muestras temporales, se calculó el promedio de abundancia y riqueza infracomunitaria (Bush et al. 1997) y la prevalencia total de parásitos (porcentaje de hospedadores con al menos un individuo parásito).

Las comparaciones de los descriptores parasitológicos entre años (1, 2 y 3 ) y estaciones (primavera, verano, otoño e invierno) fueron realizadas mediante estadística no paramétrica, dado a que la transformación de datos (en $\log _{10}$ y arcosen) no fue suficiente para su normalización, condición para el uso de estadística paramétrica. La prueba de Kruskal-Wallis de una vía fue aplicada para comparar abundancia, riqueza infracomunitaria de parásitos, así como la LT de peces, entre años y entre estaciones de muestreo. Luego de la comparación de riqueza y abundancia infracomunitaria de parásitos y la LT, se aplicó el test a posteriori de Bonferroni (Garcia et al. 20071), para determinar cuáles de los grupos comparados tuvieron diferencias significativas entre sí (Rosner 2000). Estos análisis fueron realizados con el paquete estadístico STATISTICA v 8.0 (Statsoft $2010^{2}$ ). La prevalencia total de parásitos fue comparada

${ }^{1}$ García S, M Molina, M Lozano \& F Herrera. 2007. Tests no paramétricos de comparaciones múltiples con algoritmo de control en el análisis de algoritmos evolutivos: Un caso de estudio con los resultados de la sesión especial en optimización continua CEC'2005. Actas de las I Jornadas sobre Algoritmos Evolutivos y Metaheurísticas, Zaragoza, 12 y 13 de septiembre, pp. 219-227.

${ }^{2}$ StatSoft. 2010. Electronic Statistics Textbook. StatSoft. Inc., Tulsa. <http://www.statsoft.com/textbook/> 
para cada especie de pez entre años de muestreo mediante tablas de contingencias de 2 × 3 , y entre estaciones mediante tablas de contingencia $2 \times 4$. Para esta prueba estadística, se usó el estadígrafo de Chi-cuadrado $\left(\chi^{2}\right)$ y el paquete estadístico MICROSTAT v 4.0 (Ecosoft $\left.1984^{3}\right)$.

Se aplicaron correlaciones de Spearman entre los descriptores parasitológicos, abundancia, riqueza infracomunitaria, y la longitud total del pez. Las correlaciones entre estas variables fueron realizadas para la totalidad de ejemplares por especie de pez. También se correlacionó la prevalencia total de parásitos, los valores promedios de abundancia y riqueza infracomunitaria con respecto al promedio de LT de peces por muestra temporal.

Por último, se realizó análisis discriminantes para determinar que especies parásitas eran las que contribuían con las diferencias temporales y si el tamaño corporal del hospedador era una variable importante en las variaciones comunitarias de parásitos (Clarke \& Warwick 1994). Luego, en este análisis se consideró la abundancia de parásitos en aquellas especies parásitas que tenían al menos $2 \%$ de frecuencia en cada especie de pez, lo cual permitió un mejor ajuste de los datos a una distribución normal. El nivel de significación utilizado fue de $5 \%(\alpha=0,05)$ para todos los análisis estadísticos.

\section{Resultados}

En todos los peces examinados, se encontró un total de 9.103 parásitos correspondientes a 28 taxa. Los ectoparásitos en peces residentes fueron más comunes (abundantes, frecuentes y ricos en especies), mientras que los endoparásitos lo fueron para peces temporales (Fig. 1, Anexo 1).

En Scartichthys viridis se recolectaron 6.509 parásitos pertenecientes a 23 taxa, mientras que en Helcogrammoides chilensis se recolectaron 922 parásitos correspondientes a 9 taxa. En los peces temporales, 536 parásitos se encontraron en Girella laevifrons, que pertenecían a 10 taxa, y en Sicyases sanguineus se recolectaron 1.136 parásitos correspondientes a 12 taxa. El detalle de las especies parásitas, con sus prevalencias, abundancias e intensidad promedio, se encuentra en el Anexo 1.

\section{VARIACIONES DE LAS COMUNIDADES DE PARÁSITOS}

Las comunidades de parásitos en las cuatro especies de peces variaron significativamente, en algunos de sus descriptores, entre años y estaciones (Tablas 1 y 2), aunque con patrones de variación distintos entre peces residentes y temporales. En las especies residentes, Scartichthys viridis y Helcogrammoides chilensis, las comunidades de parásitos variaron estacionalmente, con valores máximos y mínimos en los descriptores parasitológicos en cada ciclo anual (Fig. 1). Los ectoparásitos mostraron variaciones marcadas por estación, siendo claramente más abundantes, ricos y prevalentes en primavera y verano. El patrón de variación de endoparásitos también fue estacional, aunque, en comparación con los endoparásitos hubo algunos leves desfases en los valores máximos y mínimos de los descriptores parasitológicos por ciclo anual; algunos descriptores mostraron sus máximos en otoño y primavera para ciertos años (Fig. 1).

Los valores máximos y mínimos de los descriptores comunitarios son los que marcan el patrón estacional en los peces residentes (Fig. 1). A pesar que los valores máximos de abundancia, prevalencia y riqueza de parásitos, sin separación entre ecto y endoparásitos, en peces residentes ocurrieron cíclicamente entre un año y otro, éstos fueron muy distintos (Fig. 1). De hecho, hubo variaciones significativas en los valores máximos y mínimos de abundancia y riqueza infracomunitaria de parásitos en Scartichthys viridis entre años (prueba $a$ posteriori, $P<0,01)$, la cual tendió a aumentar desde el primer al tercer año de muestreo (Fig. 1). La prevalencia total de parásitos también fue distinta en sus valores máximos $\left(\chi^{2}=8,56\right.$, g.l. $\left.=2, P=0,013\right)$ y en los mínimos $\left(\chi^{2}\right.$ $=91,94$, g.l. $=2, P<0,001)$ siendo el tercer año distinto a los otros. En Helcogrammoides chilensis se encontraron máximos de abundancia infracomunitaria distintos entre años, observándose mayor abundancia de parásitos en el tercer año de muestreo, aunque similares en los valores mínimos (Fig. 1). La prevalencia total de parásitos en $H$. chilensis no fue distinta entre años (Tabla 1), sin embargo, las prevalencias máximas $\left(\chi^{2}=8,22\right.$, g.l. $\left.=2, P=0,016\right)$ y mínimas $\left(\chi^{2}=1,56\right.$, g.1. $\left.=2, P<0,001\right)$ fueron mayores en el tercer año (prueba a posteriori $P<0,001$ ). La riqueza de especies parásitas promedio no fue distinta entre años (Tabla 1).

Las comunidades de parásitos en especies de peces temporales, Girella laevifrons y Sicyases sanguineus, variaron a lo largo del tiempo, sin embargo, los cambios

${ }^{3}$ Ecosoft. 1984. An Iterative General -Purpose Statistics. Ecosoft Inc., Sacramento. 

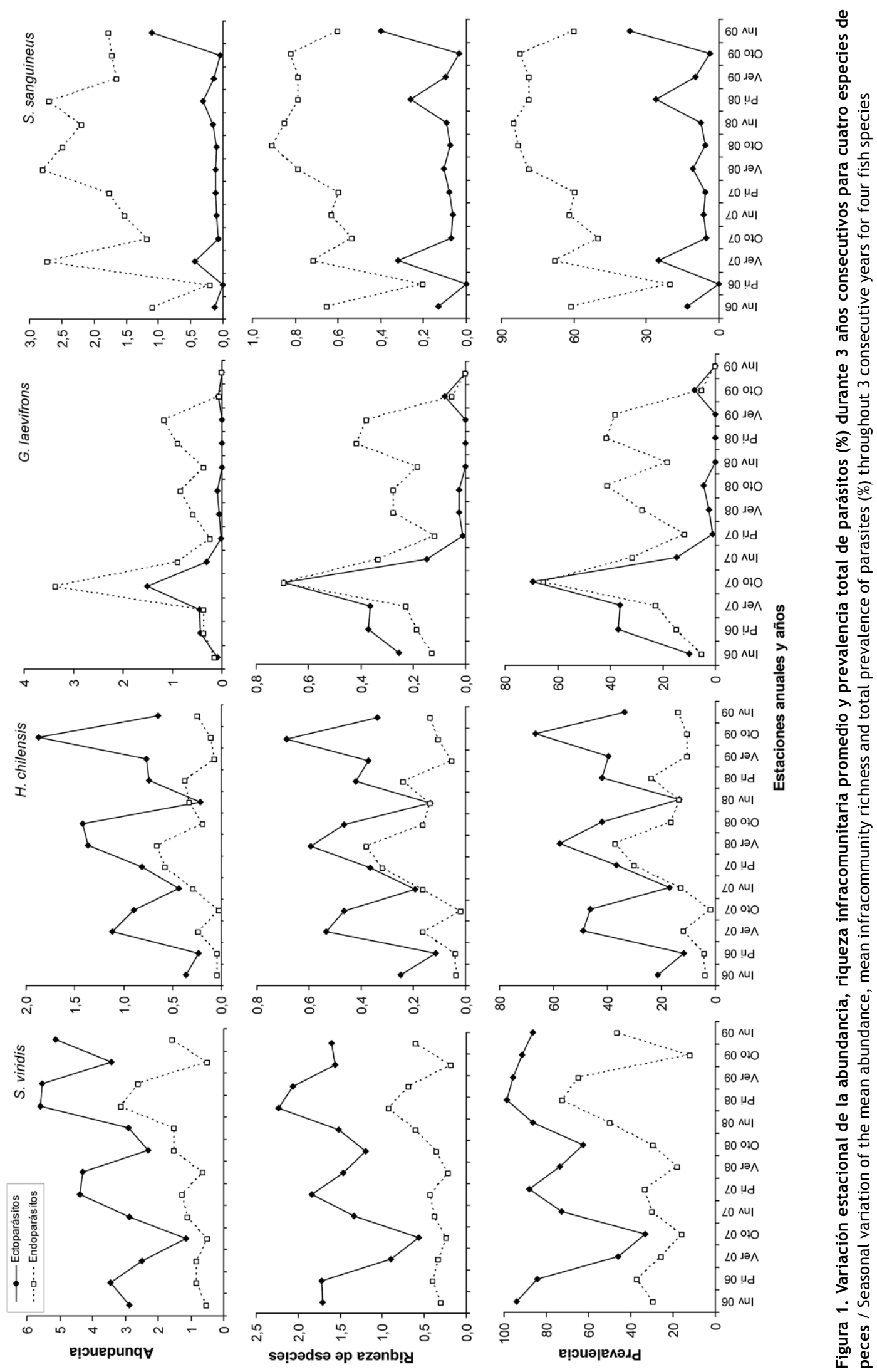

Vol. 46, №3, 2011317 
Tabla 1. Resultados del análisis estadístico de Kruskal-Wallis $(\mathrm{H})$ que compara los descriptores de abundancia total (ABU), riqueza de especies parásitas (RIQ) y Tablas de contingencia $\left(\chi^{2}\right)$ para comparaciones de prevalencia de parásitos (PRE) en cada especie hospedadora entre años de muestreo. Se incluyen resultados de pruebas a posteriori / Results of the Kruskal-Wallis statistical analysis $(\mathrm{H})$ which compares the total abundance ( $A B U)$, parasites species richness (RIQ) and Contingency Tables $\left(\chi^{2}\right)$ for the comparison of parasites prevalence (PRE) in each host species among sampling years. A posteriori test results are included

\begin{tabular}{|c|c|c|c|c|c|}
\hline \multirow[t]{2}{*}{ Especie de pez } & \multirow[t]{2}{*}{$\mathrm{N}^{\circ}$ total } & \multirow[t]{2}{*}{ Descriptor } & \multicolumn{2}{|c|}{ Entre años } & \multirow{2}{*}{$\begin{array}{c}\text { Comparaciones } \\
\text { a posteriori* }\end{array}$} \\
\hline & & & $\mathrm{H}$ & $P$ & \\
\hline \multicolumn{6}{|l|}{ Peces residentes } \\
\hline \multirow{3}{*}{ Scartichthys viridis } & 1.475 & $\mathrm{ABU}$ & $\mathrm{H}=181,17$ & $<0,001$ & Año $1 \neq$ Año $2 \neq$ Año 3 \\
\hline & & RIQ & $\mathrm{H}=143,97$ & $<0,001$ & Año $1 \neq$ Año $2 \neq$ Año 3 \\
\hline & & PRE & $\chi^{2}=208,03$ & $<0,001$ & Año $1 \neq$ Año $2 \neq$ Año 3 \\
\hline \multirow[t]{3}{*}{ Helcogrammoides chilensis } & 826 & $\mathrm{ABU}$ & $\mathrm{H}=22,16$ & $<0,001$ & Año $1=$ Año 3 \# Año 2 \\
\hline & & RIQ & $\mathrm{H}=2,89$ & 0,235 & \\
\hline & & PRE & $\chi^{2}=2,90$ & 0,234 & \\
\hline \multicolumn{6}{|l|}{ Peces temporales } \\
\hline \multirow[t]{3}{*}{ Girella laevifrons } & 500 & $\mathrm{ABU}$ & $\mathrm{H}=40,34$ & $<0,001$ & Año $1 \neq$ Año $2=$ Año 3 \\
\hline & & RIQ & $\mathrm{H}=48,69$ & $<0,001$ & Año $1=$ Año $2 \neq$ Año 3 \\
\hline & & PRE & $\chi^{2}=19,38$ & $<0,001$ & Año $1 \neq$ Año $2=$ Año 3 \\
\hline \multirow[t]{3}{*}{ Sicyases sanguineus } & 550 & $\mathrm{ABU}$ & $\mathrm{H}=23,75$ & $<0,001$ & Sólo Año 1 f Año 3 \\
\hline & & RIQ & $\mathrm{H}=18,89$ & $<0,001$ & Año $1=$ Año $2 \neq$ Año 3 \\
\hline & & PRE & $\chi^{2}=19,50$ & $<0,001$ & Año 1 = Año 2 f Año 3 \\
\hline
\end{tabular}

*Resultado de Bonferroni para abundancia y riqueza, Tabla de contingencia $2 \times 2\left(\chi^{2}\right)$ para prevalencia; $\neq$ implica diferencias estadísticas entre los grupos $(P<0,05)$

estacionales fueron menos consistentes entre años que en peces residentes. Los endoparásitos de G. laevifrons fueron más prevalentes, abundantes y ricos en otoño 2007 y 2008 (Fig. 1). El máximo de abundancia y riqueza promedio de parásitos (ecto y endoparásitos) en $G$. laevifrons fue significativamente distinto en el primer año con respecto a los otros dos años de muestreo $(P<0,001)$, lo mismo sucedió en la prevalencia máxima de parásitos $\left(\chi^{2}=16,92\right.$, g.l. $\left.=2, P=0,0002\right)$, aunque los valores más bajos fueron similares entre años $\left(\chi^{2}=0,44\right.$, g.l. $=2, P=$ $0,799)$ (Fig. 1). Sicyases sanguineus, por su parte, presentó variaciones en los descriptores parasitológicos entre años ya que el segundo año tuvo valores menores que los otros dos (Tabla 1), aunque no hubo variaciones estacionales en los descriptores parasitológicos (Tabla 2).

\section{TAMAÑo CORPORAL DE LOS PECES}

Los ejemplares capturados de Helcogrammoides chilensis y Scartichthys viridis eran juveniles y adultos con rangos de LT de 2,1-7,5 cm y 4,3-41,5 cm respectivamente. Los ejemplares de Girella laevifrons y Sicyases sanguineus eran todos juveniles con rangos de LT de 2,7-23,0 cm y 2,1-21,0 cm, respectivamente. La LT promedio de algunas especies de peces varió entre las estaciones del año. Scartichthys viridis presentó LTs significativamente menores en otoño en $\operatorname{los} 3$ años muestreados $(\mathrm{H}=182,85, P<0,001$; prueba a posteriori $P$ $<0,05)$, H. chilensis tuvo LTs significativamente distintas entre años $(\mathrm{H}=15,65, P<0,001)$ y entre estaciones $(\mathrm{H}=$ $31,87, P<0,001)$, siendo mayor en otoño. Igualmente $G$. laevifrons mostró LTs muy distintas entre años $(\mathrm{H}=51,65$, $P<0,001)$ y entre estaciones ( $\mathrm{H}=47,72, P<0,001)$, aunque sin alguna tendencia estacional o interanual (Fig. 2). Sólo S. sanguineus tuvo LTs similares entre años $(\mathrm{H}=1,54, P$ $=0,46)$ y entre estaciones $(\mathrm{H}=2,29, P=0,51)$ (Fig. 2).

La abundancia y riqueza infracomunitaria de parásitos, para el total de ejemplares de cada especie de pez, se correlacionaron positiva y significativamente con la LT de los peces de las cuatro especies consideradas $(P<$ 0,001; Tabla 3). Sin embargo, la LT corporal de los peces entre las muestras temporales mostraron diferencias significativas (entre años y estaciones), mientras que la correlación entre descriptores (abundancia y riqueza infracomunitaria promedio, y prevalencia total de parásitos en cada especie de pez) y la LT promedio de los peces por muestras temporales, no fue significativa para todas las especies hospederas (Tabla 3, Fig. 3); sólo la abundancia y riqueza promedio de parásitos de Scartichthys viridis, y la riqueza promedio y prevalencia total de parásitos aumentaron con la LT de Girella laevifrons (Tabla 3, Fig. 3). 
Tabla 2. Resultados del análisis estadístico de Kruskal-Wallis $(\mathrm{H})$ que compara los descriptores de abundancia total (ABU), riqueza de especies parásitas (RIQ) y tabla de contingencia $\left(\chi^{2}\right)$ para la prevalencia (PRE) aplicado para cada especie hospedadora entre estaciones. Se incluye resultados a posteriori. Estaciones abreviadas a las primeras 3 letras / Results of the Kruskal-Wallis statistical analysis $(\mathrm{H})$ which compares the total abundance (ABU), parasites species richness (RIQ) and Chi-square $\left(\chi^{2}\right)$ for the prevalence (PRE) in each host species among seasons. A posteriori test results are included. Seasons abbreviated to the first 3 letters

\begin{tabular}{lcrcc}
\hline Especie de pez & Descriptor & \multicolumn{2}{c}{ Entre estaciones } & Resultado a posteriori* \\
& & H & $P$ & \\
\hline Peces residentes & & & & \\
Scartichthys viridis & ABU & 155,14 & $<0,001$ & VER $=$ INV $\neq$ OTO $\neq$ PRI \\
& RIQ & 153,63 & $<0,001$ & Todas las estaciones distintas entre sí \\
& PRE & 140,31 & $<0,001$ & Todas las estaciones distintas entre sí \\
Helcogrammoides chilensis & ABU & 42,49 & $<0,001$ & INV $\neq$ otras estaciones, PRI $\neq$ VER \\
& RIQ & 41,84 & $<0,001$ & INV $\neq$ otras estaciones \\
& PRE & 49,19 & $<0,001$ & OTO $=$ PRI $\neq$ INV $\neq$ VER \\
Peces temporales & & & & \\
Girella laevifrons & ABU & 27,63 & $<0,001$ & OTO $\neq$ otras estaciones \\
& RIQ & 26,74 & $<0,001$ & OTO $\neq$ otras estaciones \\
Sicyases sanguineus & PRE & 20,77 & $<0,001$ & OTO $\neq$ PRI, INV $=$ VER \\
& ABU & 2,96 & 0,398 & \\
& RIQ & 2,78 & 0,426 & \\
& PRE & 2,49 & 0,475 & \\
\hline
\end{tabular}

* Resultado de Bonferroni para abundancia y prevalencia, $\chi^{2}$ para prevalencia; $\neq$ implica diferencias estadísticas entre los grupos $(P<0,05)$

Tabla 3. Correlación de Spearman $\left(r_{s}\right)$ y su probabilidad (P) para los descriptores parasitológicos de abundancia (ABU), riqueza (RIQ) infracomunitaria y prevalencia total (PRE) con respecto al longitud total del pez, considerando todos los datos y datos promedio por muestra temporal / Spearman correlation $\left(r_{s}\right)$ and its probability $(P)$ for the parasitological descriptors of abundance (ABU), infracommunity richness (RIQ) and total prevalence (PRE) in relation to the fish total length, taking into account the whole data set and the mean data set per seasonal sample

Todos los datos* Promedio por estaciones

\begin{tabular}{lcccccc}
\hline & & \multicolumn{2}{c}{ Todos los datos* } & \multicolumn{2}{c}{$\begin{array}{c}\text { Promedio por estaciones } \\
(\mathrm{n}=13)\end{array}$} \\
Hospedador & Descriptor & $\mathrm{r}_{\mathrm{s}}$ & $P$ & $\mathrm{r}_{\mathrm{s}}$ & $P$ \\
\hline Peces residentes & & & & & \\
Scartichthys viridis & ABU & 0,794 & $<0,001$ & 0,813 & $<0,001$ \\
& RIQ & 0,778 & $<0,001$ & 0,670 & 0,011 \\
Helcogrammoides chilensis & PRE & - & - & 0,462 & 0,107 \\
& ABU & 0,406 & $<0,001$ & 0,335 & 0,252 \\
& RIQ & 0,402 & $<0,001$ & 0,231 & 0,435 \\
Peces temporales & PRE & - & - & 0,154 & 0,603 \\
Girella laevifrons & & & & & \\
& ABU & 0,486 & $<0,001$ & 0,451 & 0,116 \\
Sicyases sanguineus & RIQ & 0,482 & $<0,001$ & 0,637 & 0,018 \\
& PRE & - & - & 0,666 & 0,012 \\
& ABU & 0,494 & $<0,001$ & 0,462 & 0,107 \\
& RIQ & 0,378 & $<0,001$ & 0,401 & 0,166 \\
& PRE & - & - & 0,300 & 0,304 \\
\hline
\end{tabular}

*Datos totales $(\mathrm{N})$ por especie en Tabla 1 


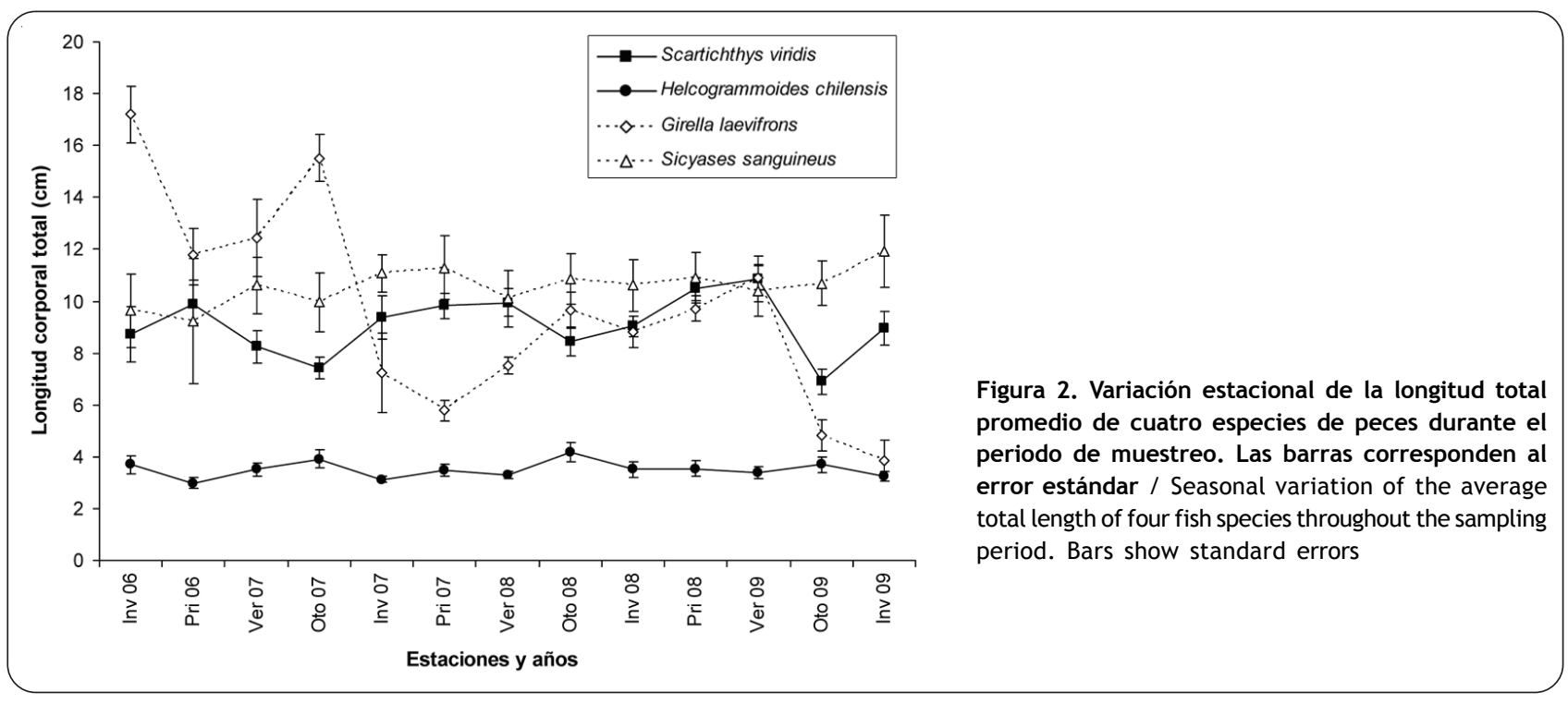

Tabla 4. Resultados del análisis discriminante para cada especie de pez y variables consideradas (longitud total del hospedador (LT) y especies parásitas), las variables están organizadas desde la más a la menos importante según el estadígrafo $\mathrm{F}$-remove / Results of the discriminant analysis per each fish species and per variable tested (fish total length (LT) and parasite species), variables are organized from the most to the least important, according to F-remove

\begin{tabular}{|c|c|c|c|c|c|c|}
\hline \multirow[t]{2}{*}{ Variables por pez } & \multicolumn{2}{|c|}{$\begin{array}{l}\text { Resultado por } \\
\text { especie de pez }\end{array}$} & \multicolumn{4}{|c|}{ Resultado por variable } \\
\hline & Wilks' & $P$ & Wilks' & Partial & F-remove & $P$ \\
\hline Scartichthys viridis & 0,551 & $<0,0001$ & & & & \\
\hline Colobomatus sp. & & & 0,646 & 0,854 & 21,32 & $<0,001$ \\
\hline LT (cm) & & & 0,611 & 0,903 & 13,40 & $<0,001$ \\
\hline Piscicolidae gen. sp. 1 & & & 0,596 & 0,925 & 10,05 & $<0,001$ \\
\hline Lecithasteridae gen. sp. & & & 0,593 & 0,930 & 9,43 & $<0,001$ \\
\hline Microcotyle sp. & & & 0,569 & 0,970 & 3,83 & $<0,001$ \\
\hline Megasolena sp. & & & 0,568 & 0,971 & 3,65 & $<0,001$ \\
\hline Lepeophtheirus zbigniewi & & & 0,564 & 0,978 & 2,78 & 0,001 \\
\hline Neobenedenia melleni & & & 0,560 & 0,984 & 1,99 & 0,021 \\
\hline Helcogrammoides chilensis & 0,668 & $<0,0001$ & & & & \\
\hline Pseudophyllidea gen. sp. & & & 0,742 & 0,901 & 7,39 & $<0,001$ \\
\hline Holobomolochus chilensis & & & 0,724 & 0,923 & 5,55 & $<0,001$ \\
\hline Tetraphyllidea gen. sp. & & & 0,692 & 0,966 & 2,37 & 0,005 \\
\hline $\mathrm{LT}(\mathrm{cm})$ & & & 0,687 & 0,974 & 1,81 & 0,043 \\
\hline Lecithaster sp. & & & 0,681 & 0,982 & 1,20 & 0,276 \\
\hline Girella laevifrons & 0,231 & $<0,0001$ & & & & \\
\hline $\mathrm{LT}(\mathrm{cm})$ & & & 0,536 & 0,431 & 52,99 & $<0,001$ \\
\hline Clavellotis dilatata & & & 0,290 & 0,798 & 10,16 & $<0,001$ \\
\hline Similascarophis chilensis & & & 0,252 & 0,916 & 3,69 & $<0,001$ \\
\hline Sicyases sanguineus & 0,768 & $<0,0001$ & & & & \\
\hline Piscicolidae gen. sp. 2 & & & 0,848 & 0,906 & 4,61 & $<0,001$ \\
\hline Proctoeces sp. & & & 0,821 & 0,936 & 3,06 & $<0,001$ \\
\hline Acanthochondria sicyases & & & 0,798 & 0,963 & 1,73 & 0,058 \\
\hline $\mathrm{LT}(\mathrm{cm})$ & & & 0,790 & 0,972 & 1,26 & 0,235 \\
\hline
\end{tabular}




\section{ANÁLISIS DISCRIMINANTES}

Los análisis discriminantes entre las muestras temporales de las cuatro especies de peces consideradas, mostraron variaciones significativas de la abundancia promedio de ciertas especies parásitas (Tabla 4). Aunque la mayoría de las especies parásitas usadas en el análisis mostraron diferencias en el tiempo, sólo unas pocas tuvieron influencia significativa en las variaciones temporales (según valores de F-remove, que mientras más alto sea, la variable es más influyente para la varianza de los datos y discriminante ente los grupos comparados). De acuerdo a esto, 3 especies parásitas de Scartichthys viridis fueron las que mostraron las mayores diferencias temporales poblacionales (Colobomatus sp., Piscicolidae gen. sp. y Lecithasteridae gen. sp., Tabla 4). En las otras especies de peces, una o dos especies parásitas variaban significativamente entre muestras temporales, Pseudophyllidea gen. sp. y Holobomolochus chilensis en Helcogrammoides chilensis, Clavellotis dilatata en Girella laevifrons y Piscicolidae gen. sp. y Proctoeces sp. en Sicyases sanguineus (Tabla 4). Del análisis discriminante además, se obtuvo que la LT de los peces fue la primera y segunda variable en importancia para las variaciones de abundancia parasítica en G. laevifrons y $S$. viridis respectivamente, mientras que la LT de los peces fue menos o nada de importante para $H$. chilensis y $S$. sanguineus respectivamente (Tabla 4).

\section{Discusión}

En este estudio se ha mostrado que las infracomunidades de parásitos de peces intermareales cambian en abundancia, riqueza y prevalencia a lo largo del año, sin embargo, sólo en peces residentes tales variaciones fueron claramente estacionales y cíclicas dentro de un año y otro. En peces temporales, por su parte, se evidenció un escaso patrón estacional en las comunidades de parásitos; se observó que algunos descriptores parasitológicos variaron, con máximos consistentes entre dos años, pero no entre los tres años muestreados.

Las diferencias observadas en la variación temporal de las comunidades de parásitos entre peces residentes y temporales pueden radicar en las distintas características ecológicas de ambos grupos de peces, considerando el tiempo de permanencia y el estadio ontogénico que presentan cuando están en la zona intermareal. Los peces residentes están presentes en las pozas intermareales durante todo el año, por lo que es posible encontrar tanto juveniles como adultos. Así, la permanencia de estos peces en un ambiente determinado por largo tiempo y la interacción constante con otras especies favorece la adquisición de parásitos, ya sea de especies ectoparásitas que se transmiten directamente al pez o de invertebrados capaces de transmitir endoparásitos. En el caso de peces temporales, las fluctuaciones de sus comunidades de parásitos puede ser una respuesta a una suma de factores; por un lado, los peces temporales considerados en este estudio habitan en el intermareal sólo en estado juvenil y presentan una corta estadía en ese ambiente. Por lo tanto, la baja adquisición de parásitos en peces jóvenes suele ser debido a que un cuerpo pequeño ofrece pocos recursos de espacio, o simplemente el hospedador no está en condiciones morfológicas adecuadas para la fijación de los parásitos; por otro lado, los peces jóvenes tardan en adquirir su defensa inmunológica frente a parásitos (Karnoven et al. 2004), lo que dependerá del tiempo de exposición y de la carga parasitaria a la cual han estado expuestos (Wakelin 1984).

Es importante destacar además, que las especies parásitas de peces intermareales residentes son más específicas que aquellas encontradas en los peces temporales (Muñoz \& Cortés 2009), lo cual solo es posible si la relación hospedador-parásito se ha desarrollado en un largo tiempo evolutivo. Si ocurre esta estrecha relación entre hospedadores y parásitos, podría ser que ambos grupos de simbiontes tengan tiempos de desarrollo sincronizados. Existen estudios, en otros sistemas parásito-hospedador, que indican que los parásitos pueden modificar sus periodos de desarrollo para su transmisión y supervivencia si las condiciones ambientales no son favorables (Calero-Torralbo \& Valera 2008). Además, los parásitos podrían ajustar sus periodos infectivos según la presencia de los hospedadores requeridos (Foster 1969, Okabe \& Makino 2008). Así, ciertas especies parásitas lograrían variar alguno de estos aspectos de acuerdo a sus posibilidades de transmisión al siguiente hospedador. Los cambios poblacionales de parásitos podrían repercutir en las características comunitarias de éstos, generando variaciones temporales que pueden cambiar entre un año y otro.

El tamaño corporal del hospedador es otro factor relevante para la carga parasitaria lo que ha sido demostrado para los peces en general (e.g., Hayward et al. 1998, Lo et al. 1998, Timi \& Poulin 2003, Muñoz \& Randhawa 2011, Muñoz \& Zamora 2011). Sin embargo, según los análisis de correlación entre las variables y los análisis discriminantes, su influencia en las comunidades de parásitos fue variable entre las especies de peces intermareales usadas en este estudio. Sólo en Scartichthys 


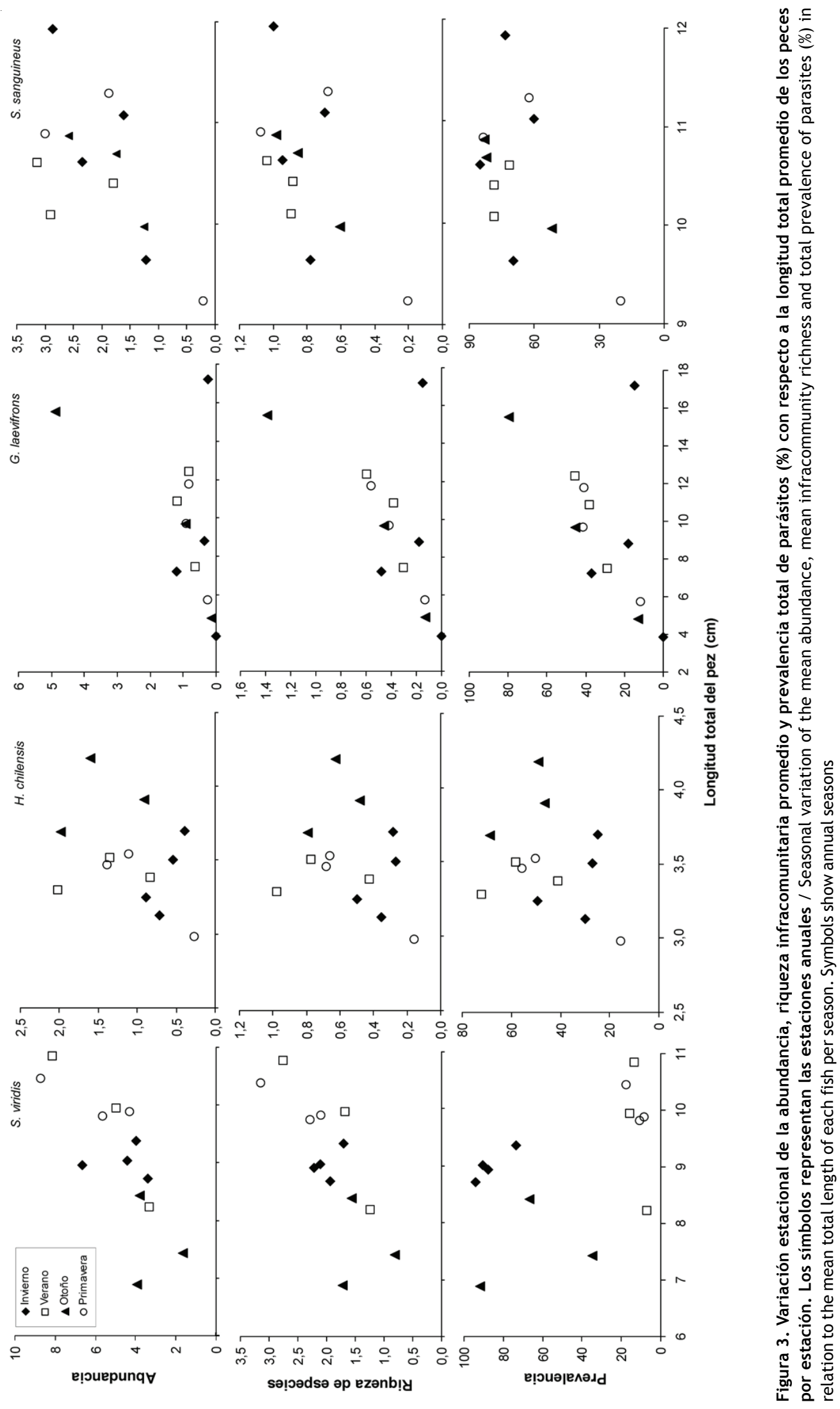


viridis hubo una alta correlación entre descriptores parasitológicos y LTs de los peces (Tabla 3, Fig. 2). El reclutamiento de juveniles de $S$. viridis en las pozas intermareales ocurre en otoño alcanzando sus mayores longitudes en primavera y verano (Fig. 3) (HernándezMiranda \& Ojeda 2006), adquiriendo constantemente nuevos parásitos, ya sea en número de especies y de individuos a lo largo del año (Muñoz \& Randhawa 2011), lo que resulta en el patrón de variación estacional tanto de las poblaciones como de las comunidades de parásitos. Por otro lado, el otro pez residente, Helcogrammoides chilensis, presentó mayores tamaños corporales y mayor abundancia, prevalencia y riqueza de parásitos en otoño (Fig. 3), sin embargo no hubo correlación significativa entre estas variables durante los muestras temporales (Tabla 3), además, en el análisis discriminante esta variable (LT) fue la cuarta en importancia para las variaciones comunitarias de parásitos, es decir tuvo cierta influencia en la variación, pero no fue la más importante. Por lo tanto, lo que determina la estacionalidad de las comunidades de parásitos en $H$. chilensis, puede estar ligada solo en parte a la LT del hospedador, mientras que, por otro lado, las características poblacionales de parásitos podrían asociarse a las variaciones de sus ciclos de vida, tiempo de desarrollo y cambios en el ambiente (e.g., Granath \& Esch 1983, Marcogliese \& Esch 1989). Con respecto a esto último, el fenómeno de El Niño comenzó a manifestarse el año $2009^{4}$, donde se realizaron los 3 últimos muestreos del presente estudio, por lo tanto, el aumento de abundancia de parásitos en peces residentes, hacia el tercer año específicamente, pudo ser afectado por los cambios en la temperatura del agua que caracteriza a este fenómeno.

La LT de peces temporales en general no tuvo correlación importante con los descriptores comunitarios de parásitos. La LT de Sicyases sanguineus, por ejemplo, no varió entre muestras temporales (Fig. 2), aunque hubo diferencias en los descriptores parasitológicos (Tablas 2, 3). En cambio, Girella laevifrons, presentó correlación entre la abundancia y riqueza de parásitos con respecto a la longitud del hospedador, pero esa variación no se relacionó con las estacionalidad (Fig. 3). Por lo tanto, en ambas especies de peces, las variaciones en las comunidades de parásitos no podrían ser atribuidas a la diferencia temporal de la LT de los hospedadores, siendo posible que algún factor ambiental tenga mayor influencia en las poblaciones y comunidades de parásitos (Zander 2003).
Cabe indicar que los parásitos también tienen sus ciclos biológicos con determinadas características, es decir, pueden tener distinto número y tipo de hospedadores (i.e., grupo taxonómico), tiempo de desarrollo, $\mathrm{y}$ a las características del medio que permitan tal desarrollo (Tucker et al. 2000). No obstante, el desarrollo de los parásitos de alguna forma es dependiente no solo de la presencia de sus hospedadores (intermediarios y definitivos) sino también de sus características biológicas (e.g., dieta, conducta, distribución), ya que en algún momento el parásito debe entrar en contacto con él hospedador para seguir su desarrollo (e.g., Mikheev et al. 2000). A este respecto, este estudio determinó que si bien la LT de los hospedadores puede relacionarse para la abundancia, prevalencia y riqueza de parásitos, en las variaciones estacionales de las comunidades de parásitos, ésta no fue la variable causal de tal patrón en todas las especies de peces. Es decir, otros factores, no definidos en este estudio, afectarían a los parásitos.

Finalmente, de esta investigación se puede concluir que los cambios en las infracomunidades de parásitos en peces intermareales son variables durante el año y entre años, aunque con un patrón de variación cíclico estacional para peces residentes, habitantes frecuentes de ese ambiente, mientras que peces temporales, que migran a otros hábitats cuando alcanzan la madurez sexual, presentan escaso patrón de variación estacional en sus comunidades de parásitos. Las variables que influyen en tales diferencias pueden ser muchas, para algunas especies el tamaño corporal de los peces fue importante para obtener la variación temporal observada, mientras que, en otras especies se pueden considerar influencias intrínsecas y extrínsecas a los parásitos, causantes de los patrones de variación temporal aquí observado. Estos resultados, permiten así indagar sobre las dinámicas poblacionales y comunitarias de parásitos, sus variaciones naturales en años normales (i.e., sin fenómenos oceanográficos significativos), y de esta forma entender mejor la dependencia de los parásitos y relación con sus hospedadores.

\section{Agradecimientos}

Se agradece la colaboración de Camila Delgadillo, Karen Flores, Paulina Moraga, Catalina Solís, Leonardo Zamora y Juan Ayala en la recolección y disección de peces durante distintas etapas de esta investigación. Este estudio fue financiado por el Proyecto FONDECYT de Iniciación 11060006.

${ }^{4}<$ http://www.ncdc.noaa.gov/sotc/enso/2009/> 


\section{LITERATURA CITADA}

Aldana M, JM Pulgar, F Ogalde \& FP Ojeda. 2002. Morphometric and parasitological evidence for ontogenetic and geographical shifts in trophic status of intertidal fishes. Bulletin of Marine Science 70: 55-74.

Bush A, K Lafferty, J Lotz \& A Shostak. 1997. Parasitology meets ecology on its own terms: Margolis et al. revisited. The Journal of Parasitology 83: 575-583.

Calero-Torralbo MA \& F Valera. 2008. Synchronization of host-parasite cycles by means of diapause: host influence and parasite response to involuntary host shifting. Parasitology 135(11):1343-1352.

Clarke K \& R Warwick. 1994. Change in marine communities: An approach to statistical analysis and interpretation, 170 pp. PRIMER-E Ltd., Plymouth.

Cortés Y \& G Muñoz. 2009. Metazoan parasites infracommunities of the toadfish Aphos porosus (Picses: Batrachoidiformes) in central Chile: how variable are they over time? The Journal of Parasitology 95(3): 753-756.

Díaz F \& M George-Nascimento. 2002. Estabilidad temporal de las infracomunidades de parásitos en la borrachilla Scartichthys viridis (Valenciennes. 1836) (Pisces: Blenniidae) en la costa central de Chile. Revista Chilena de Historia Natural 75(4): 641-649.

Fellis K \& G Esch. 2004. Community structure and seasonal dynamics of helminth parasites in Lepomis cyanellus and L. macrochirus from Charlie's pond. North Carolina: host size and species as determinants of community structure. Journal of Parasitology 90(1): 41-49.

Fernández J \& G Esch. 1991. Guild structure of larval trematodes in the snail Helisoma anceps: patterns and processes at the individual host level. The Journal of Parasitology 77: 528-539.

Flecker AS \& B Feifarek. 1994. Disturbance and the temporal variability of invertebrate assemblages in two Andean streams. Freshwater Biology 31: 131-142.

Foster MS. 1969. Synchronized life cycles in the orange crowned warbler and its mallophaganparasites. Ecology 50: 315-323.

Gibson R \& R Yoshiyama. 1999. Intertidal fish communities. En: Horn M, K Martin \& M Chotkowski (eds). Intertidal fishes: life in two worlds, pp. 264-296. Academic Press, New York.

González M \& M Oliva. 2006. Similarity and structure of the ectoparasite communities of rockfish species from the southern Chilean coast in a temporal scale. Parasitology 133: 335-343.

Granath WO Jr \& GW Esch. 1983. Temperature and other factors that regulate the composition and intrapopulation densities of Bothriocephalus acheilognathi (Cestoda) in Gambusia affinis (Pisces). The Journal Parasitology 69: 1116-1124.
Hayward CJ, KML Perera \& K Rohde. 1998. Assemblages of ectoparasites of a pelagic fish, slimy mackerel (Scomber australasicus), from south-eastern Australia. International Journal for Parasitology 28: 263-273.

Hernández-Miranda E \& FP Ojeda. 2006. Inter-annual variability of somatic growth rate and mortality of coastal fishes off central Chile: an ENSO driven process? Marine Biology 149: 925-936.

Herrmann K \& R Sorensen. 2009. Seasonal dynamics of two mortality-related trematodes using an introduced snail. The Journal of Parasitology 95(4): 823-828.

Hewitt JE, SE Thrush \& VJ Cummings. 2001. Assessing environmental impacts: effects of spatial and temporal variability at likely impact scales. Ecological Applications 11: 1502-1516.

Horn M \& R Gibson. 1988. Peces intermareales. Investigación y Ciencias 138: 50-57.

Hurtrez-Boussès S, M de Garine-Wichatitsky, P Perret, J Blondel \& F Renaud. 1999. Variations in prevalence and intensity of blow fly infestations in an insular Mediterranean population of blue tits. Canadian Journal of Zoology 77(2): 337-341.

Inostroza R, G Sievers, J Roa \& R Aguirrebeña. 1993. Prevalencia e intensidad de infección estacional por Ceratothoa gaudichaudii en salmones (Salmo salar) cultivados en agua de mar en el sur de Chile. Archivos de Medicina Veterinaria 25(2): 173-179.

Karnoven A, PJ Hudson, O Seppälä \& T Valtonen. 2004. Transmission dynamics of a trematode parasite: exposure, acquired resistance and parasite aggregation. Parasitology Research 92: 183-188.

Kim Y \& EN Powell. 2009. Effects of climate variability on interannual variation in parasites, pathologies, and physiological attributes of bivalves from the U.S. East, Gulf, and West coasts. Environmental Bioindicator 4: 6796.

Lo CM, S Morand \& R Galzin. 1998. Parasite diversity $\backslash$ host age and size relationship in three coral-reef fishes from French Polynesia. International Journal for Parasitology 28(11): 1695-1708.

Mann GF. 1954. La vida de los peces en aguas chilenas, 342 pp. Universidad de Chile, Santiago.

Marcogliese D. 2002. Food webs and the transmission of parasites to marine fish. Parasitology 124: S83-S99.

Marcogliese D \& G Esch. 1989. Alterations in seasonal dynamics in Bothriocephalus acheilognathi in a North Carolina cooling reservoir over a seven year period. The Journal of Parasitology 75: 378-382.

Mikheev V, A Mikheev, A Pasternak \& E Valtonen. 2000. Light-mediated host searching strategies in a fish ectoparasite. Argulus foliaceus L. (Crustacea: Branchiura). Parasitology 120: 409-416. 
Muñoz AA \& P Ojeda. 1998. Guild structure of carnivorous intertidal fishes of the Chilean coast: implications of ontogenetic dietary shifts. Oecologia 114: 563-573.

Muñoz G \& Y Cortés. 2009. Parasite communities of a fish assemblage from the intertidal rocky zone of central Chile: similarity and host specificity between temporal and resident fish. Parasitology 136: 1291-1303.

Muñoz G \& HS Randhawa. 2011. Monthly variation in the parasite communities of the intertidal fish Scartichthys viridis (Blenniidae) from central Chile: Are there seasonal patterns? Parasitology Research 109(1): 53-62.

Muñoz G \& L Zamora. 2011. Ontogenetic variation in parasite infracommunities of the clingfish Sicyases Sanguineus (Pisces: Gobiesocidae). The Journal of Parasitology 97(1): 14-19.

Muñoz G, F Garcías, V Valdebenito \& M GeorgeNascimento. 2001. Parasitofauna y alimentación de Notothenia c.f. angustata Hutton, 1875 (Pisces: Nototheniidae) en el intermareal de dos localidades del Golfo de Arauco, Chile. Boletín Chileno de Parasitología 56: 2933.

Muñoz G, V Valdebenito \& M George-Nascimento. 2002. La dieta y la fauna de parásitos metazoos del torito Bovichthys chilensis Regan 1914 (Pisces: Bovichthydae) en la costa de Chile centro-sur: variaciones geográficas y ontogenéticas. Revista Chilena de Historia Natural 75: 661671.

Muñoz-Muga P \& G Muñoz. 2010. Comunidades de parásitos de Scartichthys viridis (Pisces: Blenniidae) de Chile central: localidad vs. longitud del hospedador. Revista de Biología Marina y Oceanografía 45(1): 165-169.

Negovetich N \& G Esch. 2007. Long-term analysis of Charlie's pond: fecundity and trematode communities of Helisoma anceps. The Journal of Parasitology 93(6): 1311-1318.

Okabe K \& S Makino. 2008. Life cycle and sexual mode adaptations of the parasitic mite Ensliniella parasitica (Acari: Winterschmidtiidae) to its eumenine wasp host, Allodynerus delphinalis (Hymenoptera: Vespidae). Canadian Journal of Zoology 86: 470-478.
Poulin R. 2007. Evolutionary ecology of parasites, 332 pp. Princeton University Press, New Jersey.

Rosner B. 2000. Fundamentals of biostatistics, 792 pp. Duxbury Thomson Learning, Belmont.

Sandland G, C Goater \& A Danylchuk. 2001. Population dynamics of Ornithodiplostomum ptychocheilus metacercariae in fathead minnows (Pimephales promelas) from four northern Alberta lakes. The Journal of Parasitology 87: 744-748.

Timi JT \& R Poulin. 2003. Parasite community structure within and across host populations of a marine pelagic fish: How repeatable is it? International Journal for Parasitology 33: 1353-1362.

Tucker C, C Sommerville \& R Wootten. 2000. The effect of temperature and salinity on the settlement and survival of copepodids of Lepeophtheirus salmonis (Køyer. 1837) on atlantic salmon. Salmo salar L. Journal of Fish Diseases 23: 309-320.

Varas E \& FP Ojeda. 1990. Intertidal fish assemblages of the central central Chilean coast: diversity, abundance and trophic patterns. Revista de Biología Marina 25: 59-70

Wakelin D. 1984. Inmunity to parasites how animals control parasitic infections, 165 pp. Edward Arnold, London.

Yurlova N, S Vodyanitskaya, E Serbina, V Biserkov, B Georgiev \& N Chipev. 2006. Temporal variation in prevalence and abundance of metacercariae in the pulmonate snail Lymnaea stagnalis in Chany Lake. West Siberia. Russia: long-term patterns and environmental covariates. The Journal of Parasitology 92(2): 249-259.

Zander C. 2003. Four-year monitoring of parasite communities in gobiid fishes of the south-western Baltic. I. Guild and component community. Parasitology Research 90: 502-511.

Zander C, L Reimer \& K Barz. 1999. Parasite communities of the Salzhaff (Northwest Mecklenburg. Baltic Sea). I. Structure and dynamics of communities of littoral fish, especially small-sized fish. Parasitology Research 85: 356372.

Recibido el 30 de marzo de 2011 y aceptado el 1 de septiembre de 2011 
Anexo 1. Especies parásitas encontradas en cuatro especies de peces, residentes $(R)$ y temporales $(T)$ del intermareal rocoso de Chile central; número de ejemplares por especie parásita encontrados $(\mathrm{N})$, prevalencia $(\mathrm{P})$, y promedios de abundancia (A) e intensidad (I) parásita con sus respectivas desviaciones estándar ( \pm DE) / Parasite species found in two resident $(R)$ and two temporal $(T)$ fish species from the rocky intertidal of central Chile; number of individuals per parasite species found $(\mathrm{N})$, prevalence $(\mathrm{P})$, and mean abundance $(\mathrm{A})$ and intensity $(\mathrm{I})$ with their respective standard deviations $( \pm D E)$

\begin{tabular}{|c|c|c|c|c|c|}
\hline Hospedador & Parásitos & $\mathrm{N}$ & $\mathrm{P}(\%)$ & $\mathrm{A} \pm \mathrm{DE}$ & $\mathrm{I} \pm \mathrm{DE}$ \\
\hline \multirow[t]{25}{*}{ Scartichthys viridis $(\mathrm{R})$} & Ectoparásitos & & & & \\
\hline & Piscicolidae gen. sp. $1(\mathrm{H})$ & 2.179 & 45,6 & $1,48 \pm 3,10$ & $3,24 \pm 3,92$ \\
\hline & Acanthochondria sicyases $(\mathrm{C})$ & 1 & 0,1 & $<0,01 \pm 0,03$ & 1,00 \\
\hline & Lepeophtheirus zbigniewi (C) & 204 & 7,5 & $0,14 \pm 0,67$ & $1,85 \pm 1,70$ \\
\hline & Caligidae gen. sp. (C)* & 15 & 0,8 & $0,01 \pm 0,13$ & $1,25 \pm 0,87$ \\
\hline & Colobomatus sp. (C) & 1.443 & 54,9 & $0,98 \pm 1,00$ & $1,78 \pm 0,62$ \\
\hline & Holobomolochus chilensis (C) & 16 & 0,8 & $0,01 \pm 0,14$ & $1,45 \pm 0,69$ \\
\hline & Turbellaria gen. sp. & 5 & 0,2 & $0,00 \pm 0,08$ & $1,67 \pm 0,58$ \\
\hline & Microcotyle sp. (M) & 864 & 24,0 & $0,59 \pm 1,53$ & $2,44 \pm 2,28$ \\
\hline & Neobenedenia melleni (M) & 51 & 3,2 & $0,03 \pm 0,20$ & $1,09 \pm 0,28$ \\
\hline & Gyrodactylus sp. (M) $(\mathrm{n}=360)$ & & 83,6 & & \\
\hline & Endoparásitos & & & & \\
\hline & Lecithasteridae gen. sp. (D) & 1.357 & 28,3 & $0,92 \pm 2,32$ & $3,25 \pm 3,37$ \\
\hline & Helicometrina nimia $(\mathrm{D})$ & 4 & 0,2 & $<0,01 \pm 0,06$ & $1,33 \pm 0,58$ \\
\hline & Digeneo no identificado* & 1 & 0,1 & $<0,01 \pm 0,03$ & 1,00 \\
\hline & Hemiuridae gen. sp. (D)* & 1 & 0,1 & $<0,01 \pm 0,03$ & 1,00 \\
\hline & Monascus filliformis (D) & 21 & 0,7 & $0,01 \pm 0,25$ & $2,10 \pm 2,23$ \\
\hline & Hemipera sp. (D) & 58 & 1,6 & $0,04 \pm 0,47$ & $2,42 \pm 2,84$ \\
\hline & Megasolena sp. (D) & 234 & 6,4 & $0,16 \pm 0,87$ & $2,49 \pm 2,45$ \\
\hline & Pseudophyllidea gen. sp. (E)* & 1 & 0,1 & $<0,01 \pm 0,03$ & 1,00 \\
\hline & Similascarophis chilensis $(\mathrm{N})$ & 4 & 0,2 & $<0,01 \pm 0,06$ & $1,33 \pm 0,58$ \\
\hline & Spirurida gen. sp. $(\mathrm{N})$ & 4 & 0,1 & $<0,01 \pm 0,10$ & 4,00 \\
\hline & Pseudodelphis chilensis $(\mathrm{N})$ & 32 & 1,5 & $0,02 \pm 0,20$ & $1,45 \pm 0,80$ \\
\hline & Nematoda (no identificado)* & 4 & 0,1 & $<0,01 \pm 0,07$ & $2,00 \pm 0,00$ \\
\hline & Corynosoma sp. (A)* & 9 & 0,5 & $0,01 \pm 0,09$ & $1,29 \pm 0,49$ \\
\hline \multirow[t]{12}{*}{ Helcogrammoides chilensis $(\mathrm{R})$} & Ectoparásitos & & & & \\
\hline & Piscicolidae gen. sp. $1(\mathrm{H})$ & 12 & 1,3 & $0,01 \pm 0,13$ & $1,09 \pm 0,30$ \\
\hline & Holobolomochus chilensis (C) & 674 & 44,5 & $0,82 \pm 1,40$ & $2,25 \pm 1,47$ \\
\hline & Gyrodactylus sp. $(\mathrm{M})(\mathrm{n}=32)$ & n.d. & 59,4 & n.d. & n.d. \\
\hline & Endoparásitos & & & & \\
\hline & Helicometrina nimia (D) & 1 & 0,1 & $<0,01 \pm 0,03$ & 1,00 \\
\hline & Lecithaster sp. (D) & 24 & 2,6 & $0,03 \pm 0,19$ & $1,14 \pm 0,48$ \\
\hline & Digenea (no identificado)* & 1 & 0,1 & $<0,01 \pm 0,03$ & 1,00 \\
\hline & Hemipera sp. (D) & 1 & 0,1 & $<0,01 \pm 0,03$ & 1,00 \\
\hline & Tetraphyllidea gen. sp. (E)* & 35 & 2,9 & $0,04 \pm 0,29$ & $1,46 \pm 0,93$ \\
\hline & Pseudophyllidea gen. sp. (E)* & 172 & 11,0 & $0,21 \pm 0,75$ & $1,89 \pm 1,40$ \\
\hline & Corynosoma sp. (A)* & 2 & 0,2 & $<0,01 \pm 0,05$ & $1,00 \pm 0,00$ \\
\hline \multirow[t]{12}{*}{ Girella laevifrons $(\mathrm{T})$} & Ectoparásitos & & & & \\
\hline & Piscicolidae gen. sp. $1(\mathrm{H})$ & 3 & 0,6 & $0,01 \pm 0,08$ & $1,00 \pm 0,00$ \\
\hline & Lepeophtheirus zbigniewi (C) & 5 & 1 & $0,01 \pm 0,10$ & $1,00 \pm 0,00$ \\
\hline & Holobolomochus chilensis (C) & 1 & 0,2 & $<0,01 \pm 0,04$ & 1,00 \\
\hline & Clavellotis dilatata $(\mathrm{C})$ & 119 & 12,2 & $0,24 \pm 0,76$ & $1,95 \pm 1,18$ \\
\hline & Endoparásitos & & & & \\
\hline & Helicometrina nimia (D) & 1 & 0,2 & $<0,01 \pm 0,04$ & 1,00 \\
\hline & Bothriocephalidae gen. sp. (E) & 1 & 0,2 & $<0,01 \pm 0,04$ & 1,00 \\
\hline & Similascarophis chilensis $(\mathrm{N})$ & 401 & 27 & $0,80 \pm 1,98$ & $2,97 \pm 2,84$ \\
\hline & Spirurida gen. sp. $(\mathrm{N})$ & 1 & 0,2 & $<0,01 \pm 0,04$ & 1,00 \\
\hline & Nematoda (no identificado)* & 2 & 0,4 & $<0,01 \pm 0,06$ & $1,00 \pm 0,00$ \\
\hline & Corynosoma sp. (A)* & 2 & 0,4 & $<0,01 \pm 0,06$ & $1,00 \pm 0,00$ \\
\hline
\end{tabular}




\section{Continuación Anexo 1 / Annex 1 continued}

\begin{tabular}{|c|c|c|c|c|c|}
\hline Hospedador & Parásitos & $\mathrm{N}$ & $\mathrm{P}(\%)$ & $\mathrm{A} \pm \mathrm{DE}$ & $\mathrm{I} \pm \mathrm{DE}$ \\
\hline \multirow[t]{15}{*}{ Sicyases sanguineus $(\mathrm{T})$} & Ectoparásitos & & & & \\
\hline & Piscicolidae gen. sp. $1(\mathrm{H})$ & 1 & 0,2 & $<0,01 \pm 0,04$ & 1,00 \\
\hline & Piscicolidae gen. sp. $2(\mathrm{H})$ & 63 & 6,7 & $0,11 \pm 0,60$ & $1,70 \pm 1,66$ \\
\hline & Acanthochondria sicyases (C) & 39 & 5,1 & $0,07 \pm 0,34$ & $1,39 \pm 0,69$ \\
\hline & Holobomolochus chilensis (C) & 1 & 0,2 & $<0,01 \pm 0,04$ & 1,00 \\
\hline & Gyrodactylus sp. $(\mathrm{n}=8)$ & & 100,0 & & \\
\hline & Endoparásitos & & & & \\
\hline & Helicometrina nimia (D) & 39 & 1,6 & $0,07 \pm 0,90$ & $4,33 \pm 5,85$ \\
\hline & Proctoeces sp. (D) & 977 & 67,3 & $1,78 \pm 2,33$ & $2,64 \pm 2,41$ \\
\hline & Proctoeces lintoni (D) & 6 & 0,4 & $0,01 \pm 0,22$ & $3,00 \pm 2,83$ \\
\hline & Digenea (no identificado)* & 1 & 0,2 & $<0,01 \pm 0,04$ & 1,00 \\
\hline & Hemipera sp. (D) & 3 & 0,6 & $0,01 \pm 0,07$ & $1,00 \pm 0,00$ \\
\hline & Tetraphyllidea gen. sp. (E)* & 1 & 0,2 & $<0,01 \pm 0,04$ & 1,00 \\
\hline & Pseudophyllidea gen. sp. (E)* & 2 & 0,2 & $<0,01 \pm 0,09$ & 2,00 \\
\hline & Pseudodelphis chilensis $(\mathrm{N})$ & 3 & 0,4 & $0,01 \pm 0,10$ & $1,50 \pm 0,71$ \\
\hline
\end{tabular}

* parásitos larvales, n.d.: datos no disponibles; H: Hirudinea; C: Copepoda; M: Monogenea; D: Digenea, E: Eucestoda; N: Nematoda; A: Acanthocephala 